\title{
Batteriewechselstationen für Elektroautos - China setzt einen neuen Standard
}

Im Jahr 2007 wurde vom früheren SAP-Manager Shai Agassi das Unternehmen „Better Place“ gegründet. Die Idee von Agassi war, landesweit ein System von Batteriewechselstationen aufzubauen, um das Reichweitenproblem bei Elektroautos zu lösen. In weniger als zehn Minuten kann der leere Akku eines Elektroautos an einer Wechselstation mit einem vollgeladenen getauscht werden. Elektroautos wären nicht mehr durch zeitraubende Ladevorgänge gehandicapt. Das Elektroauto würde funktionieren wie ein Diesel- oder Benzin-Pkw. Eine eingängige Idee.

Trotz viel Publicity stellte sich das System als wenig marktfähig heraus. Im Jahr 2014 ging „Better Place“ in Insolvenz. Damals waren batterieelektrische Autos und das Wechselstationenmodell wenig gefragt. 8.522 vollelektrische Pkw wurden im Insolvenzjahr von "Better Place“ in Deutschland verkauft. Der Marktanteil betrug dürre 0,3\%. Noch schlechter sah die Bilanz beim Pkw-Bestand aus. Nach den Daten des Kraftfahrtbundesamts (KBA) waren damals nur 12.156 reine Elektroautos unter den 44 Mio. Pkw auf Deutschlands Straßen. Ein System von Batteriewechselstationen musste „verhungern“, sprich, ein „Henne-Ei-Problem" vereitelte den Erfolg. Mittlerweile boomt das Elektroauto. Dieselgate, die Klimakrise, die harten $\mathrm{CO}_{2}$-Auflagen der EU sowie großzügige Prämien von bis zu 9.000 Euro für den Kauf von Elektroautos haben Elektromobilität salonfähig gemacht. In den ersten sieben Monaten wurden in Deutschland 174.180 batterieelektrische Pkw-Neuwagen zugelassen, knapp $11 \%$ aller neuen Pkw. In Summe waren 417.609 vollelektrische Autos im April 2021 auf Deutschlands Straßen. Also müsste das Ei zur Lösung des „HenneEi-Problems" gelegt sein. Volumen für die Infrastrukturanbieter von Batteriewechselstationen wäre vorhanden.

(C) Der/die Autor:in 2021. Open Access: Dieser Artikel wird unter der Creative Commons Namensnennung 4.0 International Lizenz veröffentlicht (creativecommons.org/licenses/by/4.0/deed.de).

Open Access wird durch die ZBW - Leibniz-Informationszentrum Wirtschaft gefördert.

Prof. Dr. Ferdinand Dudenhöffer ist Direktor des CAR-Center Automotive Research in Duisburg.
Warum das Batteriewechselsystem nicht marktfähig ist

Tesla, aber auch die VW-Gruppe mit inrem breiten Markenportfolio, Mercedes, BMW sowie alle anderen westlichen Autobauer haben dennoch kein Interesse am Batteriewechselgeschäft. Tesla hat sehr schnell seine Schnelllader (Elon Musk nennt die natürlich Supercharger) quer über Europa aufgestellt und betreibt in China 7.000 davon. Stromkonzerne, Stadtwerke, Mineralölgesellschaften und die Autobauer selbst stellen Schnelllader auf. Noch im Juli 2021 hat das Bundesverkehrsministerium eine Ausschreibung mit hohen Zuschüssen für das Aufstellen von Schnellladern verabschiedet. Freilich erst als Ausschreibung, aber ab 2023 sollen dann nach den Plänen von Verkehrsminister Scheuer die staatlich bezuschussten Schnelllader wie Pilze aus dem Boden schießen. Hat also die Batteriewechselstation das Legen des Eies verschlafen? Wenn die eine Infrastruktur steht, ist es sehr schwer, Investor:innen von einer konkurrierenden zu überzeugen.

Hinzu kommt, dass die Ladezeiten der Elektroautos kürzer werden. Hohe Ladespannungen, bei Porsche bis 800 Volt, ermöglichen Schnellladevorgänge in weniger als 20 Minuten. Des Weiteren macht die Batterietechnik Fortschritte. Wenn die Feststoffbatterie industriell hergestellt werden kann, irgendwann zwischen 2025 und 2030 sollte das den Batterieherstellern zufolge gelingen, können die Ladevorgänge nochmals erheblich verkürzt werden. Zusätzlich steigt die Energiedichte der Batterie, sprich 1.000 km Reichweite mit einer Stromladung sind dann kein Problem mehr, und das bei deutlichen Kostensenkungen und geringerem Batteriegewicht. Die Luft für den Aufbau von Batteriewechselstationen wird noch dünner. Der Standard für die Ladeinfrastruktur ist gesetzt. Ein großes Netz an Batteriewechselstationen scheint nicht mehr vorstellbar.

Es kommt ein weiteres Manko dazu. Die Batterie ist das neue Herz des Autos, und die Leistungsfähigkeit der Batterie definiert den Wettbewerbsvorsprung des Autobauers. Tesla ist dabei, die Batterie in die Fahrzeugstruktur des Autos zu integrieren. Mit riesigen Druckgussmaschinen, die Elon Musk natürlich Giga Press nennt, wird das vordere und hintere Chassis des Fahrzeugs jeweils in einem einzigen Teil gegossen. Auch im Tesla-Werk in Berlin Grünheide werden die Gigapressen eingesetzt. Die Fahr- 
zeugkonstruktion wird so ausgelegt, dass die Batteriepacks fest mit der Fahrzeugstruktur verbunden sind. Damit kann das Elektroauto deutlich leichter und kostengünstiger gebaut werden. Diese auch Cell-to-Chassis genannte Technologie „verschweißt“, wenn man so will, das Fahrzeug mit seinem Batteriepack. Damit fält es noch schwerer, sich einen Marktdurchbruch für Batteriewechselsysteme vorzustellen.

Weiter gilt, dass es die Batterie nicht gibt. Auf der einen Seite werden unterschiedliche Zellformate verbaut. Größere Zellenformate, wie die sogenannte Blade Battery des chinesischen Autobauers BYD oder bei Tesla die 4680 Zelle, erlauben Kostenvorteile bei weniger Gewicht und Batterievolumen. Zusätzlich sind Zellen, selbst im gleichen Format, oft deutlich differenziert. So nutzt Tesla für die Einstiegsvariante des Model 3 Zellen mit Lithium-Eisenphosphat-Kathoden. In höherwertigen Modellen werden Kobalt-Nickel-Kathoden oder Kobalt-Nickel-Mangan-Verbindungen genutzt. Da die Batteriezellentwicklung mit großen Innovationssprüngen voranschreitet, werden Zellen aus dem Jahr 2020 nicht mehr mit Zellen des Jahres 2025 identisch sein. Es wird deutlich, dass nicht nur die immer besser ausgebaute Ladeinfrastruktur, einer Infant Industry mit einem Batteriewechselsystem den Markteintritt schwermacht. Eine Wechselstationinfrastruktur braucht hohe Standardisierungen um eine „Einheitsbatterie“. Große Vielfalt an unterschiedlichen Batterietypen auf Lager zu halten, zerstört die Ökonomie eines Batteriewechselstationssystems. Last, not least gibt es heute kein westliches Autobauunternehmen, das seine Elektroautos so konstruiert, dass ein Batteriepack in kurzer Zeit gewechselt werden kann. Als Zwischenfazit lässt sich festhalten: Unter „normalen" Marktgesetzen haben Batteriewechselsysteme keine Chance zum Durchbruch.

\section{Königsmacher und Innovationstreiber China}

China ist mit Abstand der größte Automarkt der Welt und das wird sich in den nächsten 100 Jahren nicht ändern. 2020 wurden in China 20 Mio. Pkw verkauft, in den USA 15 Mio., in Europa, definiert als EU28 plus EFTA, 12 Mio. Während die Märkte USA und Europa gesättigt sind mit 845 bzw. 540 Pkw pro 1.000 Einwohner:innen entfallen in China 110 Pkw auf 1.000 Einwohner:innen. Der Hunger nach Autos ist in China noch groß. Für 2030 kann man mit einem Automarkt von mehr als 30 Mio. Pkw-Neuwagen und einem chinesischen Weltmarktanteil von $32 \%$ rechnen. Der Markt für vollelektrische $\mathrm{Pkw}$ entwickelt sich in China sehr dynamisch (vgl. Abbildung 1). Gemäß dem 14. Fünfjahresplan der chinesischen Regierung von 2020 sollen 2025 in China 20\% der Neuwagen als NEV (New Energy Vehicles = Plug-In und vollelektrisch) verkauft werden.

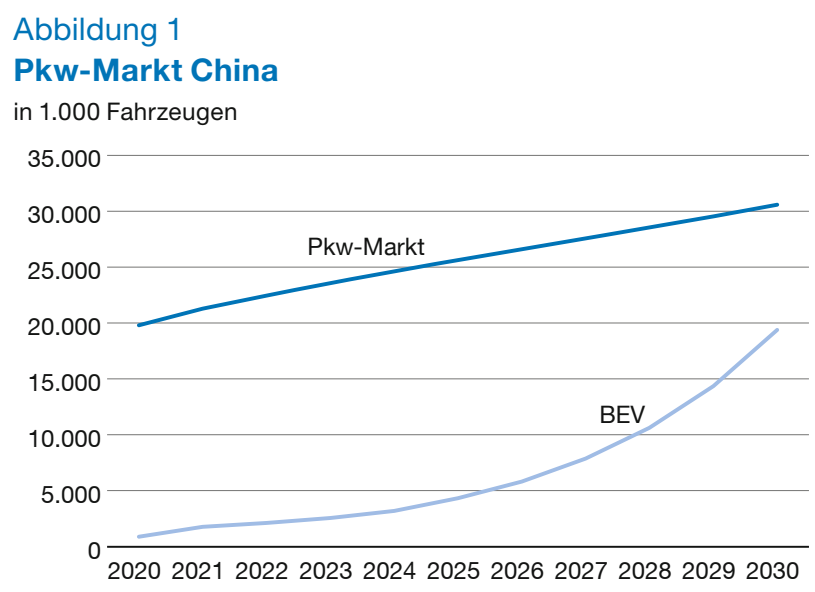

Anmerkungen: BEV = Elektroauto mit Batterie; 2020 Ist-Wert; ab 2021 Prognose von CAR, Duisburg.

Quelle: CAR

Nach unserer Einschätzung wird der überwiegende Teil vollelektrisch sein. Wenn also einer einen Technologiewechsel durchsetzen kann, dann ist es China. China ist durch seinen riesigen Binnenmarkt Königsmacher. China definiert den Standard.

China stellt aber nicht nur auf die Größe des Binnenmarkts ab, sondern will weltweit technologieführend werden. Das ist ein klares, von der kommunistischen Partei Chinas definiertes Ziel. Die Voraussetzungen, um dieses Ziel zu erreichen, sind nicht nur im Automobilsektor äuBerst gut. Die größten Batteriehersteller sitzen in China, die Innovationen auf dem Batteriesektor kommen immer stärker von dort und weniger vom bisherigen Technologieführer Korea. Neben der Elektromobilität ist die zweite, noch größere Revolution der Branche das Software-Defined Car, also alles, was um autonom fahrende Autos gestaltbar ist. China hat heute schon Datenhoheit mit Weltmarktführern wie Huawei beim 5G-Internet und rollt das autonome Fahren breit aus. Damit können Batteriewechselstationen so aufgebaut werden, dass sie im kontinuierlichen Kontakt mit den Elektroautos stehen und effiziente Batterielagerbestände an den Wechselstationen vorgehalten werden. Zusätzlich werden Stromnetze - besonders in den riesigen Metropolen Chinas - besser optimiert, denn Wechselstationen können antizyklisch Batterien laden und Strom bei Nachfragespitzen in die Netze geben. Natürlich hat ein China-Standard, der dann später von der Welt übernommen wird, große Vorteile für die Unternehmen des Landes. Die westlichen Autobauer wären gezwungen, entweder in zwei Welten zu leben oder ihre Fahrzeugarchitekturen an den China-Standard anzupassen. Beides sind kostenträchtige Vorhaben. Ein Batteriewechselsystem hätte für China durchaus Charme. 


\section{China definiert den Infrastrukturstandard}

Die ersten Batteriewechselstationen in China gehen auf 2016 zurück. Die Beijing Automotive Group (BAIC Group), einer der großen Autokonzerne des Landes mit Sitz und enger Verbindung zur Stadtregierung Beijing, stellte derzeit Batteriewechselstationen in der Hauptstadt vor. In erster Linie sollten die Stationen Taxiunternehmen erlauben, Elektrotaxen ohne lange Ladezeiten zu betreiben und Elektrotaxen in Metropolen schneller zum Durchbruch zu verhelfen. Seit 2021 ist BAIC in einer Kooperation mit dem chinesischen Internetriesen BAIDU dabei, Robotertaxen in Beijing zu testen. Die Kombination zwischen Elektrotaxen und Robotaxen besitzt Innovationspotenzial und könnte zum Durchbruch völlig neuer Mobilitätssysteme in Großstädten führen. Ein weltweit riesiges Geschäftsvolumen würde sich für den First Mover China auftun. Wechselstationen kombiniert mit Robotertaxen geht deutlich weiter als die bekannten Konzepte von Ride Hailing Dienstleistern, wie etwa UBER. Mit dem Wechselstationennetz können Robocars ohne lange Ladezeiten 24 Stunden im Dienst sein und damit zu besserer Luftqualität, weniger Raumbedarf für Autos sowie hoher Kapazitätsauslastung bei Mobilitätsanbietern beitragen. Mit einem Netz von Batteriewechselstationen legt China die Grundlagen für ein Infrastrukturprojekt, das chinesischen Autobauern und Mobilitätsdienstleistenden Wettbewerbsvorteile sichert.

Der nächste Impuls für das Wechselsystem kam vom jungen chinesischen Autobauer NIO. NIO definiert die Wechselstation als Kernelement seiner Produktstrategie. Das erste Auto des Start-ups, der NIO ES8, wurde 2017 mit Batteriewechselstation vorgestellt. Im Juli 2021 war NIO nach eigenen Angaben mit 301 Wechselstationen zweitgrößter Wechselstationenbetreiber hinter dem chinesischen Unternehmen Aulton. Bis 2025 plant NIO weltweit 4.000 Wechselstationen, 1.000 davon außerhalb Chinas. Die erste NIO-Station außerhalb Chinas geht Ende 2021 in Oslo in Betrieb. Fünf weitere sollen 2022 in Großstädten Norwegens folgen. Sämtliche Fahrzeuge von NIO sind so konstruiert, dass sie einen schnellen Batteriewechsel durchführen können. An einem noch größeren Coup arbeitet der staatliche chinesische Mineralölkonzern Sinopec gemeinsam mit dem chinesischen Batteriewechselstationsbetreiber Aulton. So sollen an 30.000 Tankstellen von Sinopec Batteriewechselstationen von Aulton betrieben werden. Vollautomatische Batteriewechsel sind nach Unternehmensinformationen in einer Minute möglich.

Die Bilanz der Wechselstationen im Land der Mitte kann sich sehen lassen. Im Juni 2021 waren 716 Wechselstationen in Betrieb. Bereits im April 2020 haben Regierungsbehörden mitgeteilt, dass Elektroautos über einer
Preisgrenze von 300.000 Yuan (rund 42.000 US-\$) nicht mehr unter das staatliche Prämienprogramm fallen, es sei denn, es handele sich um Fahrzeuge mit austauschbaren Batterien. Damit sollen dem Finanzministerium zufolge innovative Geschäftsmodelle gefördert werden. Im Mai 2020 hatte Chinas Regierung Batteriewechselstationen in den "Neuen Infrastrukturplan" aufgenommen. Nahezu alle chinesischen Autobauer sind dabei, ihre Elektroautos auf das Batteriewechselsystem auszurichten. Einer der vielen Schritte dazu war eine Vereinbarung des Wechselstationsbetreibers Aulton mit 14 Autobauern, darunter die großen Gruppen FAW, BAIC, Geely, SAIC, Changan Automobile und Dongfeng Motor.

\section{Wie umgehen mit dem Phänomen „Größe setzt den Standard"?}

China setzt in hohem Tempo einen neuen Infrastrukturstandard für Elektromobilität. Weder in Europa noch in den USA sind ähnliche Projekte beobachtbar. Ähnlich wie bei Lithium-Ionen-Batterien hat China mit dem Wechselstationenmodell die Chance, klare Wettbewerbsvorteile für das Land und seine Unternehmen aufzubauen. Wie bei Lithium-Ionen-Batterien wird es lange dauern bis der EUKommission oder Bundesregierung auffält, dass wir hinterherlaufen. Die Geschichte vom Silicon Valley mit Amazon, Facebook, Google, Tesla wiederholt sich, nur diesmal unter chinesischer Flagge. Zwei Möglichkeiten bieten sich, mit dem Phänomen „Größe setzt den Standard“ umzugehen. Erstens, durch die Schaffung eigener Größe und einer Gegenmacht, so wie es US-Präsident Joe Biden in der Allianz gegen China fordert: Ein Konfrontationsmodell und die Spaltung in zwei Welten. Betrachtet man die bisherigen Erfolge der EU28-1 bei der Umsetzung von Innovationen tritt eher Ernüchterung auf. Die zweite Möglichkeit bietet das Kooperationsmodell. Es wird nicht einfach, aber die Autoindustrie hat gezeigt, dass es möglich ist. $40 \%$ der Umsätze der deutschen Autobauer kommen aus China. Kooperation auf ökonomischem Feld zeigt Erfolg. Bleibt die Frage der Werte, das Umgehen mit der Hongkong- oder UigurenFrage. Vielleicht sollten wir uns dazu die Einschätzung des früheren Bundeskanzlers Helmut Schmidt zu eigen machen: „Es gibt Werte, an denen wir absolut festhalten sollten, aber ich bin absolut nicht der Meinung, dass wir diese Werte anderen Leuten beizubringen haben.“

\section{Literatur}

China Automotive News (diverse Artikel), https://www.autonews.com/topic/china-home-page (7. September 2021).

China Daily (2021), Chinese EV maker NIO's battery-swap stations, superchargers depart for Norway, https://www.chinadaily.com. cn/a/202107/09/WS60e7e908a310efa1bd660df3.html (9. Juli 2021).

Dudenhöffer F. (2021), Eine Allianz gegen China gefährdet unsere Zukunft, Gastkommentar, Handelsblatt, 31.8.2021, 48. 